\title{
"Dark matter of genome" in cancer
}

\begin{abstract}
Cancer is a complex disease involved defects in hundreds of genes and multiple errors in cell intra- and extracellular networks. There are more than 100 types of tumors. Every type of cells in every tissue of the body may be changed to abnormal cell growth. What is the actual trigger for cancer? Genetic alterations in tumor suppressor genes, mutations or amplifications in oncogene genes (and MYC-containing dmin) can influence on metabolic pathways, proteome imbalances and cause the development of neoplasms. ${ }^{1-6}$ Defective DNA replication can result in genomic instability as DNA alterations, chromosomal rearrangements, aneuploidy, and gene amplifications which associated with tumors. ${ }^{7}$ Chromosomal instability (CIN) is subtype of genomic instability that cause the defects in chromosomal organization and segregation. ${ }^{8}$ How "junk" DNA may be functionally involved in tumorigenesis?
\end{abstract}

Keywords: genomic instability, "junk" DNA, "dark matter of genome", repetitive sequences, non-coding transcripts, transposable elements
Volume 10 Issue I - 2019

\section{Tamara Lushnikova}

Department of Pathology and Microbiology, University of Nebraska Medical Center, USA

Correspondence: Tamara Lushnikova, Department of Pathology and Microbiology, University of Nebraska Medical Center, 986495 Nebraska Medical Center, Omaha, NE 681986495, USA, Email tamlushni@gmail.com

Received: September 20, 2017| Published: February 14, 2019

\section{Introduction}

\section{Genomic instability is a hallmark of a cancer due to defects in repair genes}

Since late 60-th it is known that the most of genomic DNA of eukaryotic organisms consists from non-coding proteins sequences, various repetitive sequences. Later among repetitive and non-coding "junk" DNA or "dark matter of genome" retrotransposons were found, short interspersed nuclear elements SINEs or long short interspersed nuclear elements and LINE, ${ }^{9}$ gene controlling elements. For many years the scientists were focused on DNA sequences of genes, a small portion of genome coding proteins. Carcinogen aflatoxin $\mathrm{B}_{1}$ covalently binding to DNA showed that DNA repair was deficient in repetitive satellite DNA compare in bulk DNA despite of the initial level of modification was the same in both DNAs. ${ }^{10}$ Microsatellite mutations associate with cancers and aging. ${ }^{11}$ Tumor suppressor p53 is multifunctional transcription factor, "the guardian of the genome", the most frequently mutated gene in tumors contributes in silencing of repeats and noncoding RNAs. The p53 protein mediates expression $\sim 1000$ genes and transcription of the repeats and ncRNAs. ${ }^{12}$ Many p53 binding sites reside in transposable repeats. ${ }^{13}$ Mouse oncogene $\mathrm{Mdm}^{2}$ is a negative regulator of p53 and DNA repair genes, is overexpressed in tumors and contributes to chromosomal breaks (double-strand breaks, DSBs) and CIN at higher rate in transgenic $\mathrm{Mdm}^{2}$ mice with aging than in wild-type mice. ${ }^{14}$ The DNA sequences associated with chromosomal breaks and rearrangements are not well understood. The DNA breaks are formed as mistakes in DNA replication, transposition of mobile elements or by environmental agents. DSBs are random but some of them occurred on fragile sites what are near telomere or centromere. The precise genome-mapping of DSBs in human chromosomes revealed non-random fragmentation and DSB hot spots. ${ }^{15}$

\section{Conclusion}

Healing of DSBs by a retrotransposon was discussed in addition to two known mechanisms of DNA repair, homology directed repair (HDR) and non-homologous end-joining (NHEJ) despite that mobile elements are viewed as genetic parasites. ${ }^{16}$ Transcript-templated repair of DNA double-strand breaks is possible when RNA transcripts are used as templates for HDR. ${ }^{17}$ The advances in sequencing technology and the Encyclopedia of DNA Elements (ENCODE) highlighted the "dark matter of genome" non-coding transcripts (ncRNAs: miRNAs, piRNAs, siRNAs ) in funding of the new functional regulatory regions. ${ }^{18}$ Bioinformatics allows to detect transposable elements transcripts from RNA-seq data for characterization of novel insertions from DNA-seq data from the Cancer Genome Atlas (TCGA) what matched normal and primary tumor samples. Several families of transposable elements in human genome are active in tumorigenesis causing numerous insertion mutations. ${ }^{19}$

\section{Acknowledgments}

None.

\section{Conflicts of interest}

None.

\section{References}

1. Knuutila S, Aalto Y, Autio K, et al. DNA copy number losses in human neoplasms. Am J Pathol. 1999;155(3):683-694.

2. Sakari Knuutila, Anna-Maria Bjorkqvist, Kirsi Autio, et al. DNA Copy Number Amplifications in Human Neoplasms. Am J Pathol. 1998;152(5):1107-1123.

3. Lushnikova T, Knuutila S. DNA copy number changes in epithelioid sarcoma and its variants: a comparative genomic hybridization study. Mod Pathol. 2000;13(10):1092-1096.

4. B P Kopnin. Targets of Oncogenes and Tumor Suppressors: Key for Understanding Basic Mechanisms of Carcinogenesis. Biochemistry (Mosc). 2000;65(1):2-27.

5. Clelia Tiziana Storlazzi, Thoas Fioretos, Cecilia Surace, et al. MYCcontaining double minutes in hematologic malignancies: evidence in favor of the episome model and exclusion of MYC as the target gene. Hum Mol Genet. 2006 ;15(6):933-942.

6. Z Shaukat, D Liu, A Choo, et al. Chromosomal instability causes sensitivity to metabolic stress. Oncogene. 2015;34(31):4044-4055.

7. Wallace MD, Southard TL, Schimenti KJ, et al. Role of DNA damage response pathways in preventing carcinogenesis caused by intrinsicreplication stress. Oncogene. 2014;33(28):3688-3695. 
8. Laura A Díaz-Martínez, Juan F Giménez-Abián, Duncan J Clarke. Chromosome cohesion - rings, knots, orcs and fellowship. J Cell Sci. 2008;121(Pt 13):2107-2114

9. Wagstaff BJ, Kroutter EN, Derbes RS, et al. Molecular Reconstruction of Extinct LINE-1 Elements and Their Interaction with Nonautonomous Elements. Mol Biol Evol. 2013;30(1):88-99.

10. S A Leadon, M E Zolan, P C Hanawalt. Restricted repair of aflatoxin B1 induced damage in alpha DNA of monkey cells. Nucleic Acids Res. 1983;11(16):5675-5689.

11. RJC Slebos, M Li, S Vadivelu1, et al. Microsatellite mutations in buccal cells are associated with aging and head and neck carcinoma. $\mathrm{Br} J$ Cancer. 2008;98(3):619-626.

12. Leonova KI, Brodsky L, Lipchick B, et al. p53 cooperates with DNA methylation and a suicidal interferon response to maintain epigenetic silencing of repeats and noncoding RNAs. Proc Natl Acad Sci US A. 2013;110(1):E89-98.

13. Feng Cui, Michael V Sirotin, Victor B Zhurkin. Impact of Alu repeats on the evolution of human p53 binding sites. Biology Direct. 2011;6:2.
14. Lushnikova T, Bouska A, Odvody J, et al. Aging mice have increased chromosome instability that is exacerbated by elevated Mdm 2 expression. Oncogene. 2011;30(46):4622-4631.

15. Tchurikov NA, Kretova OV, Fedoseeva DM, et al. DNA double-strand breaks coupled with PARP1 and HNRNPA2B1 binding sites flank coordinatelyexpressed domains in human chromosomes. PLoS Genet. 2013;9(4):e1003429.

16. Garfinkel DJ. Genetic loose change: how retroelements and reverse transcriptase heal broken chromosomes. Trends Microbiol. 1997;5(5):173-175.

17. Trott DA, Porter AC. Hypothesis: transcript-templated repair of DNA double-strand breaks. Bioessays. 2006;28(1):78-83.

18. Di Mauro V, Catalucci D. The importance of being ncRNAs: from bit players as "junk DNA" to rising stars on the stage of the pharmaceutical industry. Ann Transl Med. 2017;5(6):147.

19. Clayton EA, Wang L, Rishishwar L, et al. Patterns of Transposable Element Expression and Insertion in Cancer. Front Mol Biosci. 2016;3:76. 